CMU-HEP94-17

DOE-ER/40682-71

hep-ph/9406306

May, 1994

\title{
A MODEL FOR THE ORIGIN AND MECHANISMS OF CP VIOLATION
}

\author{
YUE-LIANG WU \\ Department of Physics, \\ Carnegie-Mellon University, \\ Pittsburgh, Pennsylvania 15213, U.S.A.
}

Invited Talk at the 5th Conference on the Intersections of Particle and Nuclear Physics May 31-June 6, 1994, at St. Petersburg, Florida To appear in the Proceedings, ed. S.J. Seestrom, (AIP, 1994) 
CMU-HEP94-17

DOE-ER/40682-71

hep-ph/9406306

May, 1994

\title{
A Model for the Origin and Mechanisms of CP Violation
}

\author{
Yue-Liang Wu \\ Department of Physics, Carnegie-Mellon University, \\ Pittsburgh, Pennsylvania 15213, U.S.A.
}

\begin{abstract}
In this talk I will show that the two-Higgs doublet model with vacuum $\mathrm{CP}$ violation and approximate global $U(1)$ family symmetries may provide one of the simplest and most attractive models for understanding the origin and mechanisms of $\mathrm{CP}$ violation. It is shown that the mechanism of spontaneous symmetry breaking provides not only a mechanism for generating masses of the bosons and fermions, but also a mechanism for creating $\mathrm{CP}$-phases of the bosons and fermions, so that $\mathrm{CP}$ violation occurs, after spontaneous symmetry breaking, in all possible ways from a single CP phase of the vacuum and is generally classified into four types of CP-violating mechanism. A new type of CP-violating mechanism in the charged Higgs boson interactions of the fermions is emphasized and can provide a consistent description for both established and reported $\mathrm{CP}-, \mathrm{P}$ - and T-violating phenomena. Of particular importance is the new source of $\mathrm{CP}$ violation for charged Higgs boson interactions that lead to the value of $\epsilon^{\prime} / \epsilon$ as large as $10^{-3}$ independend of the CKM phase.
\end{abstract}




\section{BASIC ASSUMPTIONS}

The origin and mechanisms of $\mathrm{CP}$ violation have been investigated for thirty years since the discovery of $\mathrm{CP}$ violation. In this talk I will present the most recent work [1, 2] about new sources of CP violation. I will show how the origin and mechanisms may be understood in the framework of $S U(2) \times U(1)$ gauge theory with two-Higgs doublets. The model is built based on the following two assumptions:

(1) CP violation originates solely from a single CP-violating phase of the vaccum.

(2) The model exhibits Approximate Global U(1) Family Symmetries (AGUFS) which act only on the fermions.

The first assumption is introduced to understand the origin of $\mathrm{CP}$ violation. The second assumption is motivated from the smallness of the Cabibbo-Kobayash-Maskawa quark mixing angles and the observed suppression of the flavor-changing neutral currents.

\section{VACUUM CP VIOLATION}

In order to prevent the so-called domain-wall problem from arising explicitly due to spontaneous $\mathrm{CP}$ violation, we observe the following fact that

In the gauge theories of spontaneous symmetry breaking, CP violation can be originated solely from the vacuum after spontaneous symmetry breaking, even if CP symmetry is not good prior to the symmetry breaking. The prerequisite condition for such a statement is: CP nonconservation occurs only at one term in the Higgs potential. This requirement actually results that the vacuum must violate CP symmetry. In particular, this condition may be simply realized by imposing an universal rule on the whole lagrangian. That is, in a renormalizable lagrangian all the interactions with dimension-four conserve $\mathrm{CP}$ and only interactions with dimension-two exhibit CP nonconservation. It may also be naturally implemented through imposing some symmetries. For convenience, we refer such a CP violation as a Vacuum CP Violation (VCPV).

Let me illustrate the simplest case with imposing the universal rule stated above, the Higgs potential for the two Higgs doublets can be simply written in the following general 
form

$$
\begin{aligned}
V(\phi)= & \lambda_{1}\left(\phi_{1}^{\dagger} \phi_{1}-\frac{1}{2} v_{1}^{2}\right)^{2}+\lambda_{2}\left(\phi_{2}^{\dagger} \phi_{2}-\frac{1}{2} v_{1}^{2}\right)^{2} \\
& +\lambda_{3}\left(\phi_{1}^{\dagger} \phi_{1}-\frac{1}{2} v_{1}^{2}\right)\left(\phi_{2}^{\dagger} \phi_{2}-\frac{1}{2} v_{2}^{2}\right)+\lambda_{4}\left[\left(\phi_{1}^{\dagger} \phi_{1}\right)\left(\phi_{2}^{\dagger} \phi_{2}\right)-\left(\phi_{1}^{\dagger} \phi_{2}\right)\left(\phi_{2}^{\dagger} \phi_{1}\right)\right] \\
& +\frac{1}{2} \lambda_{5}\left(\phi_{1}^{\dagger} \phi_{2}+\phi_{2}^{\dagger} \phi_{1}-v_{1} v_{2} \cos \delta\right)^{2}+\lambda_{6}\left(\phi_{1}^{\dagger} \phi_{2}-\phi_{2}^{\dagger} \phi_{1}-v_{1} v_{2} \sin \delta\right)^{2} \\
& +\left[\lambda_{7}\left(\phi_{1}^{\dagger} \phi_{1}-\frac{1}{2} v_{1}^{2}\right)+\lambda_{8}\left(\phi_{2}^{\dagger} \phi_{2}-\frac{1}{2} v_{2}^{2}\right)\right]\left[\phi_{1}^{\dagger} \phi_{2}+\phi_{2}^{\dagger} \phi_{1}-v_{1} v_{2} \cos \delta\right]
\end{aligned}
$$

where the $\lambda_{i}(i=1, \cdots, 8)$ are all real parameters. If all the $\lambda_{i}$ are non-negative, the minimum of the potential then occurs at

$$
<\phi_{1}^{0}>=\frac{v_{1}}{\sqrt{2}} e^{i \delta} \equiv \frac{v}{\sqrt{2}} \cos \beta e^{i \delta}, \quad<\phi_{2}^{0}>=\frac{v_{2}}{\sqrt{2}} \equiv \frac{v}{\sqrt{2}} \sin \beta
$$

It is clear that in the above potential CP nonconservation can only occur through the vacuum, namely $\delta \neq 0$. Obviously, such a CP violation appears to be explicit in the potential when $\lambda_{6} \neq 0$, so that the domain-wall problem does not explicitly arise. Note that if $\lambda_{6}=0$, CP can still be violated spontaneously[3]. In general, one can also require one of other terms, such as the term $\lambda_{5}$ or $\lambda_{7}$ or $\lambda_{8}$, to be complex in the most general potential.

\section{APPROXIMATE GLOBAL U(1) FAMILY SYMME- TRY}

To see why assuming approximate global U(1) family symmetries (AGUFS) rather than approximate flavor symmetries, we start with a general Yukawa interaction

$$
L_{Y}=\bar{q}_{L} \Gamma_{D}^{a} D_{R} \phi_{a}+\bar{q}_{L} \Gamma_{U}^{a} U_{R} \bar{\phi}_{a}+\bar{l}_{L} \Gamma_{E}^{a} E_{R} \phi_{a}+H . C .
$$

where $q_{L}^{i}, l_{L}^{i}$ and $\phi_{a}$ are $S U(2)_{L}$ doublet quarks, leptons and Higgs bosons, while $U_{R}^{i}, D_{R}^{i}$ and $E_{R}^{i}$ are $S U(2)_{L}$ singlets. $i=1, \cdots, n_{F}$ is a family label and $a=1, \cdots, n_{H}$ is a Higgs doublet label. $\Gamma_{F}^{a}(F=U, D, E)$ are the arbitrary real Yukawa coupling matrices and parameterized in the following general form

$$
\Gamma_{F}^{a}=O_{L}^{F} \sum_{i, j=1}^{n_{F}}\left\{\omega_{i}\left(g_{a}^{F_{i}} \delta_{i j}+\zeta_{F} \sqrt{g^{F_{i}}} S_{a}^{F} \sqrt{g^{F_{j}}}\right) \omega_{j}\right\}\left(O_{R}^{F}\right)^{T}
$$


where the first term in the brackets is a diagonal matrix and the second term is an off-diagonal matrix. To facilitate a comparison between the elements of the two parts, $g^{F_{i}}$ is taken to be $g^{F_{i}}=\left|\sum_{a} g_{a}^{F_{i}} \hat{v}_{a}\right| /\left(\sum_{a}\left|\hat{v}_{a}\right|^{2}\right)^{\frac{1}{2}} .\left\{\omega_{i}, i=1, \cdots, n_{F}\right\}$ the set of diagonalized projection matrices $\left(\omega_{i}\right)_{j j^{\prime}}=\delta_{j i} \delta_{j^{\prime} i} . \quad \hat{v}_{a} \equiv<\phi_{a}^{0}>\left(a=1, \cdots, n_{H}\right)$ are Vacuum Expectation Values (VEV's) which will develop from the Higgs bosons after spontaneous symmetry breaking. $g_{a}^{F_{i}}$ are the arbitrary real Yukawa coupling constants. $S_{a}^{F}\left(a \neq n_{H}\right)$ are the arbitrary off-diagonal real matrices (i.e. $\left.\left(S_{a}^{F}\right)_{i i} \equiv 0\right)$. By convention, we choose $S_{a}^{F}=0$ for $a=n_{H}$ to eliminate the dependent parameters. $\zeta_{F}$ is a conventional parameter introduced to scale the off-diagonal matrix elements so that $\left(S_{1}^{F}\right)_{12} \equiv 1$. $\left(S_{a}^{F}\right)_{i j}$ are expected to be of order unity (some elements of $S_{a}^{F}$ may be off by a factor of 2 or more). $O_{L, R}^{F}$ are the arbitrary orthogonal matrices. Note that the above parameterization is general but very useful and powerful for our purposes in analysing various interesting physical phenomena. In general, one can always choose, by a redifinition of the fermions, a basis so that $O_{L}^{F}=O_{R}^{F} \equiv O^{F}$ (and $O^{U}=1$ or $O^{D}=1$ if it needs) as well as $O^{E}=1$ since the neutrinos are considered to be massless in this model

In the gauge interactions of the fermions, there exist large global (chiral) flavor symmetries $U(3)_{L}^{q} \times U(3)_{R}^{U} \times U(3)_{R}^{D} \times U(3)_{L}^{l} \times U(3)_{R}^{E}$. These symmetries have to be broken down to the global (vector-like) family symmetries as long as the scalar interactions of the fermions are introduced to the model. This is because the scalar interactions of the fermions change the chirality of the fermions. The resulted global family symmetries depend on the physical considerations. To see this, let us consider several special cases: Suppose that the masses of all the up-type quarks, and also the down-type quarks as well as the leptons, are equal, and there are no quark mixings and also no flavor-changing neutral scalar interactions, i.e., $g_{a}^{F_{i}}=g_{a}^{F_{j}}, O^{U}=O^{D}=1$ and $\zeta_{F}=0$, then the above large global (chiral) flavor symmetries will be broken down to the large global (vector-like) family symmetries $U(3)^{q} \times U(3)^{l}$. This symmetry violation must be large as the top quark is heavy and its Yukawa coupling is of order unity. It is known that all the fermions have different masses, suppose that the quark mixings and the flavor-changing neutral scalar interactions are still zero, thus the large global family symmetries are further broken down to the global $\mathrm{U}(1)$ family symmetries $U(1)_{1}^{q} \times U(1)_{2}^{q} \times U(1)_{3}^{q} \times U(1)_{1}^{l} \times U(1)_{2}^{l} \times U(1)_{3}^{l}$, one for each family. Due to the large mass splitting among the fermions, for instance, $m_{t} / m_{u} \sim 3 \times 10^{4}$, the symmetry violation should also be large. In the realistic case, the quarks do mix and there exist flavor-changing neu- 
tral currents, therefore the global U(1) family symmetries must be completely broken down. Nevertheless, the CKM matrix is known to deviate only slightly from unity and the observed flavor-changing neutral currents are strongly suppressed, so that at the electroweak scale any successful model will exhibit approximate global $U(1)$ family symmetries (AGUFS). In other words, the AGUFS are sufficient for a natural suppression of family-changing currents (for both charged and neutral currents). Explicitly, AGUFS indicate that

$$
\left(O^{F}\right)_{i j}^{2} \ll 1, \quad i \neq j ; \quad \zeta_{F}^{2} \ll 1
$$

where $O^{F}$ describe the AGUFS in the charged currents and $\zeta_{F}$ mainly characterizes the AGUFS in the neutral currents.

It should be noted that our considerations are unlike the Hall and Weinberg's [4] approximate global U(1) flavor symmetries, i.e., one for each fermion type. This is because we do not specify the violation of global $\mathrm{U}(1)$ symmetries to the mass parameters in our approximate global $U(1)$ family symmetries (AGUFS) ( one for each family).

\section{CP-VIOLATING INTERACTIONS}

With the above general analyses and assumptions, let me now present a detailed description for the model with vacuum $\mathrm{CP}$ violation (VCPV) and approximate global U(1) family symmetries (AGUFS). The physical interactions are usually given in the mass basis of the particles. For the simplest two-Higgs doublet model, the physical basis after spontaneous symmetry breaking is defined through $f_{L}=\left(O_{L}^{F} V_{L}^{f}\right)^{\dagger} F_{L}$ and $f_{R}=\left(O_{R}^{F} P^{f} V_{R}^{f}\right)^{\dagger} F_{R}$ with $V_{L, R}^{f}$ being unitary matrices and introduced to diagonalize the mass matrices

$$
\left(V_{L}^{f}\right)^{\dagger}\left(\sum_{i} m_{f_{i}}^{o} \omega_{i}+\zeta_{F} c_{\beta} \sum_{i, j} \sqrt{m_{f_{i}}^{o}} \omega_{i} S_{1}^{F} \omega_{j} \sqrt{m_{f_{j}}^{o}} e^{i \sigma_{f}\left(\delta-\delta_{f_{j}}\right)}\right) V_{R}^{f}=\sum_{i} m_{f_{i}} \omega_{i}
$$

with $m_{f_{i}}$ the masses of the physical states $f_{i}=u_{i}, d_{i}, e_{i}$. Where $m_{f_{i}}^{o}$ and $\delta_{f_{i}}$ are defined via

$$
\left(c_{\beta} g_{1}^{F_{i}} e^{i \sigma_{f} \delta}+s_{\beta} g_{2}^{F_{i}}\right) v \equiv \sqrt{2} m_{f_{i}}^{o} e^{i \sigma_{f} \delta_{f_{i}}}
$$

with $v^{2}=v_{1}^{2}+v_{2}^{2}=\left(\sqrt{2} G_{F}\right)^{-1}, c_{\beta} \equiv \cos \beta=v_{1} / v$ and $s_{\beta} \equiv \sin \beta=v_{2} / v$. $P_{i j}^{f}=e^{i \sigma_{f} \delta_{f_{i}}} \delta_{i j}$, with $\sigma_{f}=+$, for $f=d, e$, and $\sigma_{f}=-$, for $f=u$.

For convenience, we fix the phase convention by writing 


$$
V_{L, R}^{f} \equiv 1+\zeta_{F} c_{\beta} T_{L, R}^{f}
$$

In a good approximation, to the first order in $\zeta_{F}$ and the lowest order in $m_{f_{i}} / m_{f_{j}}$ with $i<j$, we find that $m_{f_{i}}^{2} \simeq\left(m_{f_{i}}^{o}\right)^{2}+O\left(\zeta_{F}^{2}\right)$ and for $i<j$

$$
\begin{aligned}
& \left(T_{L}^{f}\right)_{i j} \simeq-\left(T_{L}^{f}\right)_{j i}^{*} \simeq \sqrt{\frac{m_{f_{i}}}{m_{f_{j}}}}\left(S_{1}^{F}\right)_{i j} e^{-i \sigma_{f}\left(\delta-\delta_{f_{j}}\right)}+O\left(\left(\frac{m_{f_{i}}}{m_{f_{j}}}\right)^{3 / 2}, \zeta_{F}\right) \\
& \left(T_{R}^{f}\right)_{i j} \simeq-\left(T_{R}^{f}\right)_{j i}^{*} \simeq \sqrt{\frac{m_{f_{i}}}{m_{f_{j}}}}\left(S_{1}^{F}\right)_{j i} e^{-i \sigma_{f}\left(\delta-\delta_{f_{j}}\right)}+O\left(\left(\frac{m_{f_{i}}}{m_{f_{j}}}\right)^{3 / 2}, \zeta_{F}\right)
\end{aligned}
$$

With this phase convention, the CKM matrix $V$ has the following form

$$
V=\left(V_{L}^{U}\right)^{\dagger}\left(O_{L}^{U}\right)^{T} O_{L}^{D} V_{L}^{D} \equiv V^{o}+V^{\prime}
$$

where $V^{o} \equiv\left(O_{L}^{U}\right)^{T} O_{L}^{D}$ is a real matrix and $V^{\prime} \simeq \zeta_{D} c_{\beta}\left[V^{o} T_{L}^{d}\right]+\zeta_{U} c_{\beta}\left[V^{o T} T_{L}^{u}\right]^{\dagger}$ is a complex matrix. It is obvious that when $\zeta_{F} \rightarrow 0$, then $V^{\prime} \rightarrow 0$, the CKM matrix is almost described by the real matrix $V^{o}$.

The scalar interactions of the fermions read in the physical basis

$$
\begin{aligned}
L_{Y}^{I}= & \left(2 \sqrt{2} G_{F}\right)^{1 / 2} \sum_{i, j, j^{\prime}}^{3} H^{+}\left\{\bar{u}_{L}^{i} V_{i j^{\prime}}\left(m_{d_{j^{\prime}}} \xi_{d_{j^{\prime}}} \delta_{j^{\prime} j}+\frac{\zeta_{D}}{s_{\beta}} \sqrt{m_{d_{j^{\prime}}} m_{d_{j}}} S_{j^{\prime} j}^{d}\right) d_{R}^{j}-H^{-} \bar{d}_{L}^{i} V_{i j^{\prime}}^{\dagger}\right. \\
& \left.\cdot\left(m_{u_{j^{\prime}}} \xi_{u_{j^{\prime}}} \delta_{j^{\prime} j}+\frac{\zeta_{U}}{s_{\beta}} \sqrt{m_{u_{j^{\prime}}} m_{u_{j}}} S_{j^{\prime} j}^{u}\right) u_{R}^{j}+H^{+} \bar{\nu}_{L}^{i}\left(m_{e_{i}} \xi_{e_{i}} \delta_{i j}+\frac{\zeta_{E}}{s_{\beta}} \sqrt{m_{e_{i}} m_{e_{j}}} S_{i j}^{e}\right) e_{R}^{j}\right\} \\
& +\left(\sqrt{2} G_{F}\right)^{1 / 2} \sum_{i, j}^{3} \sum_{k}^{3} H_{k}^{0}\left\{\bar{u}_{L}^{i}\left(m_{u_{i}} \eta_{u_{i}}^{(k)} \delta_{i j}+\frac{\zeta_{U}}{s_{\beta}} \sqrt{m_{u_{i}} m_{u_{j}}} S_{k, i j}^{u}\right) u_{R}^{j}+\bar{d}_{L}^{i}\left(m_{d_{i}} \eta_{d_{i}}^{(k)} \delta_{i j}\right.\right. \\
& \left.\left.+\frac{\zeta_{D}}{s_{\beta}} \sqrt{m_{d_{i}} m_{d_{j}}} S_{k, i j}^{d}\right) d_{R}^{j}+\bar{e}_{L}^{i}\left(m_{e_{i}} \eta_{e_{i}}^{(k)} \delta_{i j}+\frac{\zeta_{E}}{s_{\beta}} \sqrt{m_{e_{i}} m_{e_{j}}} S_{k, i j}^{e}\right) e_{R}^{j}\right\}+ \text { H.C. }
\end{aligned}
$$

with (in the above good approximations)

$$
\begin{aligned}
& \xi_{f_{i}} \simeq \frac{\sin \delta_{f_{i}}}{s_{\beta} c_{\beta} \sin \delta} e^{i \sigma_{f}\left(\delta-\delta_{f_{i}}\right)}-\cot \beta, \\
& S_{i j}^{f} \simeq\left(e^{i \sigma_{f}\left(\delta-\delta_{f_{j}}\right)}-\frac{\sin \delta_{f_{j}}}{\sin \delta}\right)\left(S_{1}^{F}\right)_{i j}, \quad S_{j i}^{f} \simeq\left(e^{i \sigma_{f}\left(\delta-\delta_{f_{i}}\right)}-\frac{\sin \delta_{f_{j}}}{\sin \delta}\right)\left(S_{1}^{F}\right)_{j i} . \\
& \eta_{f_{i}}^{(k)}=O_{2 k}^{H}+\left(O_{1 k}^{H}+i \sigma_{f} O_{3 k}^{H}\right) \xi_{f_{i}} ; \quad S_{k, i j}^{f}=\left(O_{1 k}^{H}+i \sigma_{f} O_{3 k}^{H}\right) S_{i j}^{f} .
\end{aligned}
$$

where $O_{i j}^{H}$ is the orthogonal matrix introduced to redefine the three neutral scalars $\hat{H}_{k}^{0} \equiv$ $\left(R, \hat{H}^{0}, I\right)$ into their mass eigenstates $H_{k}^{0} \equiv(h, H, A)$, i.e. $\hat{H}_{k}^{0}=O_{k l}^{H} H_{l}^{0}$ with $(R+i I) / \sqrt{2}=$ 
$s_{\beta} \phi_{1}^{0} e^{-i \delta}-c_{\beta} \phi_{2}^{0}$ and $\left(v+\hat{H}^{0}+i G^{0}\right) / \sqrt{2}=c_{\beta} \phi_{1}^{0} e^{-i \delta}+s_{\beta} \phi_{2}^{0}$. Here $H_{2}^{0} \equiv H^{0}$ plays the role of the Higgs boson in the standard model. $H^{ \pm}$are the charged scalar pair with $H^{ \pm}=$ $s_{\beta} \phi_{1}^{ \pm} e^{-i \delta}-c_{\beta} \phi_{2}^{ \pm}$.

\section{ORIGIN AND MECHANISMS OF CP VIOLATION}

By explicitly giving the new CP-violating interactions, we see that if the relative phase between the two VEV's is nonzero, each fermion $f_{i}$ and neutral scalar $H_{k}^{0}$ are then characterized not only by their physical masses $m_{f_{i}}$ and $m_{H_{k}^{0}}$ but also by their physical phases

$$
\delta_{f_{i}} \equiv \arg \left(\left(c_{\beta} g_{1}^{F_{i}} e^{i \sigma_{f} \delta}+s_{\beta} g_{2}^{F_{i}}\right) v\right) \sigma_{f} \quad \delta_{H_{k}^{0}} \equiv \arg \left(O_{1 k}^{H}+i \sigma_{f} O_{3 k}^{H}\right)
$$

This implies that the Higgs mechanism provides not only a mechanism for generating the masses of the bosons and fermions, but also a mechanism for creating CP-phases of the bosons and fermions.

With the above sources of $\mathrm{CP}$ violation, $\mathrm{CP}$ violation occurs in all possible ways. All these vacuum-induced $\mathrm{CP}$ violations can be classified into four types of mechanism according to their interactions:

Type-I. The new type of CP-violating mechanism which arises from the induced complex diagonal Yukawa couplings $\xi_{f_{i}}$. Such a CP violation can occur through both charged- and neutral-scalar exchanges.

Type-II. Flavor-Changing Superweak -type mechanism due to the flavor-changing scalar interactions. This type of mechanism also occurs through both charged- and neutral-scalar exchanges and is described by the complex coupling matrices $S_{i j}^{f}$ in this model.

Type-III. The induced KM-type CP-violating mechanism which is characterized in this model by the complex parameters $\zeta_{F} T_{L}^{f}$ and occurs in the both charged gauge boson and charged scalar interactions of the quarks.

Type-IV. The Scalar-Pseudoscalar Mixing mechanism which is described by the mixing matrix $O_{k l}^{H}$ and the phases $\arg \left(O_{1 k}^{H}+i \sigma_{f} O_{3 k}^{H}\right)$. This type of CP violation appears in the purely bosonic interactions and also in the neutral scalar interactions of the fermions in this model. In general, such kind of scalar mixing mechanism can also occur in the charged scalar 
interactions of the fermions when there exist more than two charged scalars, for example, the Weinberg three-Higgs doublet model.

\section{PHENOMENOLOGY OF CP VIOLATION}

After understanding the origin and mechanisms of CP violation, let me now briefly discuss their main features and summarize the most interesting physical phenomena (for systematic analyses and detailed calculations see [1]).

1) Without making any additional assumptions, $m_{f_{i}}, V_{i j}, \delta_{f_{i}}\left(\right.$ or $\left.\xi_{f_{i}}\right), \delta, \tan \beta, \zeta_{F},\left(S_{1}^{F}\right)_{i j}$ (or $S_{i j}^{f}$ ), $m_{H_{k}^{0}}, m_{H^{+}}$and $O_{k l}^{H}$ are in principle all the free parameters and will be determined only by the experiments. Nevertheless, from the AGUFS, we can draw the general features that $V_{i j}^{2} \ll 1$ for $i \neq j$ and $\zeta_{F}^{2} \ll 1$. The $m_{f_{i}}$ and $V_{i j}$ already appear in the standard model and have been extensively investigated. For the other parameters, it is expected that $\left(S_{1}^{F}\right)_{i j}$ are of order unity. Moreover, in order to have the flavor-changing neutral scalar interactions (FCNSI) be suppressed manifestly, it is in favor of having $\tan \beta>1$ and $\left|\sin \delta_{f_{j}} / \sin \delta\right| \leq 1$. The diagonal Yukawa couplings $\eta_{f_{i}}^{(k)}$ or $\xi_{f_{i}}$ can be, for the light fermions, much larger than one (the corresponding couplings are equal to one in the standard model) and may, of course, also be smaller than one (this case appears to occur for heavy top quark). Nevertheless, the former case is more attractive since it will result in significant interesting phenomenological effects. The most interesting choice for large $\xi_{f_{i}}$ is $\tan \beta \gg 1$ since it favors the suppression of the flavor-changing neutral scale interactions (FCNSI).

We would like to point out that the phase convention of the CKM matrix is nontrivial in this model due to the existence of the flavor-changing neutral scalar interactions (FCNSI). Therefore, before discussing contributions to CP violating observables from various mechanisms, one should first specify the phase convention.

2) From the established $K^{0}-\bar{K}^{0}$ and $B^{0}-\bar{B}^{0}$ mixings, we obtain that $\zeta_{D} / s_{\beta}<$ $10^{-3} m_{H_{k}^{0}} / \mathrm{GeV}$. From the current experimental bound of the $D^{0}-\bar{D}^{0}$ mixing, i.e. $\Delta m_{D}<$ $1.3 \times 10^{-4} \mathrm{eV}$, we have $\zeta_{U} / s_{\beta}<3 \times 10^{-3} m_{H_{k}^{0}} / G e V$. Note that this can only be regarded as an order-of-magnitude estimation since in obtaining these values we have used the vacuum insertion approximation for the evaluation of the hadronic matrix elements. From the established CP-violating parameter $\epsilon$, it requires either to fine-tune the parameters $\delta_{d}, \delta_{s}$, 
$\arg \left(O_{1 k}^{H}+i O_{3 k}^{H}\right),\left(S_{1}^{D}\right)_{12}$ etc., so that the effective CP-phase is of order $10^{-2}$ or to choose $\zeta_{D} / s_{\beta}<10^{-4} m_{H_{k}^{0}} / G e V$. For the latter case, the effective CP-violating phases are indeed generically of order unity.

3) To see how the various mechanisms play the role on $\mathrm{CP}$ violation and provide interesting physical phenomena, let us consider the following three cases:

(i) when $\zeta_{F} / s_{\beta} \ll 1,\left(S_{1}^{F}\right)_{i j}=O(1), m_{H^{+}} \leq v=246 \mathrm{GeV}$ and $\left|\xi_{f_{i}}\right| \gg 1\left(f_{i} \neq t\right)$, it is obvious that only the new type of CP-violating mechanism (type-I) plays an important role. The effects from the flavor-changing superweak -type and KM-type mechanisms are negligible.

In this case, the CP-violating parameter $\epsilon$ can be fitted from the contributions of the short-distance box graph with charged scalar exchanges and the long-distance dispersive through the $\pi, \eta$ and $\eta^{\prime}$. This is easily achieved in our model through choosing appropriate parameters $\operatorname{Im}\left(\xi_{s} \xi_{c}\right)^{2}$ and $\operatorname{Im}\left(\xi_{s} \xi_{c}\right)$ for a given mass $m_{H^{+}}$. The ratio $\epsilon^{\prime} / \epsilon$ could be of order $10^{-3}$ from both the short-distance contribution at tree level through the charged scalar exchange and the long-distance contribution induced by the charged scalar penguin diagram. The short-distance contribution to $\epsilon^{\prime} / \epsilon$ depends on the parameters $\operatorname{Im}\left(\xi_{s} \xi_{d}^{*}\right)$ and $\operatorname{Im}\left(\xi_{s} \xi_{u}\right)$. The neutron EDM $d_{n}$ can be consistently accommodated by choosing other independent parameters, such as $\operatorname{Im}\left(\xi_{d} \xi_{c}\right)$ and $\operatorname{Im}\left(\eta_{d}^{(k)}-\eta_{u}^{(k)}\right)^{2}$ for the one-loop contribution with charged scalar and neutral scalar exchanges respectively, and $\operatorname{Im}\left(\xi_{t} \xi_{b}\right)$ and $\operatorname{Im}\left(\eta_{t}^{(k)}\right)^{2}$ for the Weinberg gluonic operator contribution with charged scalar and neutral scalar exchanges respectively, as well as $\operatorname{Im}\left(\xi_{t} \xi_{q}\right)(q=d, s, u)$ for the quark gluonic chromo-EDM. Its value is not far below to the present experimental bound and could be of order $10^{-26} \mathrm{e} \mathrm{cm}$. The electon EDM $d_{e}$ from Barr-Zee two-loop mechanism is expected to be in the present experimental sensitivity for appropriate values of $\operatorname{Im}\left(\eta_{t}^{(k)} \eta_{e}^{(k)}\right)$ and $\operatorname{Im}\left(\eta_{t}^{(k)} \eta_{e}^{(k) *}\right)$ as well as $O_{2 k}^{H} \operatorname{Im} \eta_{e}^{(k)}$. CP violation in the hyperon decay can also be significant in this case. For example, the CP asymmetry observable $A\left(\Xi^{-} \rightarrow \Lambda \pi^{-}\right) \sim 10^{-4}$ which is comparable to or even larger than in the standard model. Direct CP violation in B-meson decay is, however, small in this limit case since the phases of the mixing mass matrix and the amplitudes are suppressed either by the mass ratio $m_{b} m_{d} / m_{t}^{2}$ or by $\zeta_{F}$. Nevertheless, T-odd and CP-odd triple-product correlations could be relatively large in the inclusive and exclusive semileptonic decays of B-meson into the $\tau$ leptons. 
In addition, by including the new contributions to the neutral meson mixings from the box diagrams with charged-scalar exchange, the mass difference $\Delta m_{K}$ could be reproduced by a purely short-distance analysis when $\left|\xi_{c}\right| \gg 1$. For example, for $B_{K}=0.7$, and $m_{H^{+}}=100$ $\mathrm{GeV}$, it needs $\left|\xi_{c}\right| \simeq 13$. When $\left|\xi_{t}\right| \sim 1, B^{0}-\bar{B}^{0}$ and $B_{s}^{0}-\bar{B}_{s}^{0}$ mixings could also receive a contribution as large as in the standard model.

For more explicit, let me present a simple numerical example with the following scenario:

$$
\zeta_{F}<10^{-3}, \quad \tan \beta=\frac{v_{2}}{v_{1}} \gg 1, \quad\left|\frac{\sin \delta_{f_{i}}}{\sin \delta}\right|=O(1)
$$

with $f_{i}=u, d, s, c$, thus

$$
\xi_{f_{i}} \rightarrow \frac{\sin \delta_{f_{i}}}{\sin \delta} e^{i \sigma_{f}\left(\delta-\delta_{f_{i}}\right)} \tan \beta, \quad\left|\xi_{f_{i}}\right| \sim \tan \beta
$$

This can be easily achieved by taking

$$
\delta \sim \frac{\pi}{3}, \quad \delta_{s} \sim \delta_{d} \sim \frac{\pi}{4} \quad \delta_{u} \sim \frac{\pi}{2}+\frac{\pi}{4}, \quad \delta_{c} \sim \frac{\pi}{2}+\frac{\pi}{4}-\frac{\pi}{50}
$$

which lead to

$$
\operatorname{Im}\left(\xi_{c} \xi_{s}\right) \sim \operatorname{Im}\left(\xi_{u} \xi_{s}\right) \sim \tan ^{2} \beta, \quad \operatorname{Im}\left(\xi_{c} \xi_{s}\right)^{2} \sim \frac{1}{8} \tan ^{4} \beta, \quad \operatorname{Im}\left(\xi_{d}^{*} \xi_{s}\right) \sim 0
$$

as long as

$$
\tan \beta \sim 10\left(m_{H^{+}} / 200 G e V\right)
$$

we have

$$
\begin{aligned}
|\epsilon|=2.27 \times 10^{-3} \quad \frac{\epsilon^{\prime}}{\epsilon} & \sim(1-3) \times 10^{-3} \\
\phi\left(\psi K_{S}\right) \sim 10^{-2}, \quad A\left(\Xi^{-} \rightarrow \Lambda \pi^{-}\right) & \sim 10^{-4}, \quad d_{n} \sim 10^{-26} \mathrm{e} \mathrm{cm}
\end{aligned}
$$

This simple numerical example shows that the new type of CP-violating mechanism could be indeed significant.

(ii) when $10^{-4} m_{H_{k}^{0}} / \mathrm{GeV}<\zeta_{D} / s_{\beta}<0.1, \zeta_{U} / s_{\beta}<0.3$ for $m_{t}=175 \mathrm{GeV},\left(S_{1}^{F}\right)_{i j}=O(1)$ and $\left|\xi_{f_{i}}\right|<1$, both the new type of CP-violating mechanism and the induced KM-type mechanism become less important and the parameter $\epsilon$ is then accounted for by the flavorchanging superweak-type mechanism (type-II) together with the type-IV. If the CP-violating phases are indeed generically of order unity, thus the ratio $\epsilon^{\prime} / \epsilon$ becomes unobservably small 
$\left(\sim 10^{-6}\right)$. In this case, its effects in the B-system are also small. Whereas if $B^{0}-\bar{B}^{0}$ and $B_{s}^{0}-\bar{B}_{s}^{0}$ mixings receive large contributions from the flavor-changing neutral scalar interactions, any value of CP asymmetry in B-system can occur [6].

(iii) when $\zeta_{D} / s_{\beta} \sim 0.2$ and $\zeta_{U} / s_{\beta} \sim 0.6$ for $m_{t}=175 \mathrm{GeV}, c_{\beta} \sim s_{\beta},\left|\xi_{i}\right| \sim 1$ and $m_{H_{k}^{0}} \gg v=246 \mathrm{GeV}$, i.e., neutral scalars are very heavy, then the CP-violating mechanism is governed by the induced KM-type mechanism. But it could be still different from the standard KM-model if the charged scalar is not so heavy, this is because the new contributions from diagrams with charged-scalar exchange can be significant. Therefore only when the charged scalar also become very heavy, the induced KM-type mechanism then coincides with the standard KM-model which have been extensively studied [5].

In general, four types of CP-violating mechanism may simultaneously play an important role for the CP-violating phenomena.

\section{CONCLUSIONS}

In conclusion, it is seen that precisely measuring the direct $\mathrm{CP}$ violation in kaon (and hyperon) decays and the direct CP violation in B-system as well as the EDM's of the electron and the neutron are very important for clarifying the origin and mechanisms of $\mathrm{CP}$ violation. For instance, if the direct $\mathrm{CP}$ violation in kaon decay is large and confirmed to be of order $10^{-3}$, the neutron and electron EDMs are also in the present observable level, while direct CP violation in B-system is smaller than the standard model prediction, it then clearly indicates that the new type of CP-violating mechanism will be important.

Based on the assumption of the approximate global U(1) family symmetries (AGUFS), the mass of the scalars could be less constrained from the indirect experimental data, such as neutral meson mixings and inclusive bottom quark decays $b \rightarrow s \gamma$. Searching for these exotic scalars is worthwhile at both $e^{+} e^{-}$and hadron colliders. It is believed that the mechanisms of $\mathrm{CP}$ violation discussed in this model should also play an important role for understanding the baryogenesis at the electroweak scale. In particular, its requirement for relatively light Higgs bosons in order to avoid the wash out problem is in favor of our model. All these considerations and new features suggest that if one Higgs doublet is necessary for the generation of the masses of the bosons and fermions, then two Higgs doublets are 
needed for the origin and phenomenology of $\mathrm{CP}$ violation and also for baryogenesis at the electroweak scale.

This work was supported by DOE grant \# DE-FG02-91ER40682.

\section{References}

[1] Y.L. Wu, "A Model of CP Violation", CMU Report, CMU-HEP94-01, hep-ph/9404241, 1994; "Origin and Mechanisms of CP Violation", CMU-HEP94-02, hep-ph/9404271, 1994.

[2] Y.L. Wu and L. Wolfenstein, "Sources of CP Violation in the Two-Higgs Doublet Model", CMU-HEP94-18, DOE-ER/40682-72, 1994.

[3] T.D. Lee, Phys. Rev. D8, 1226 (1973); Phys. Rep. 9, 143 (1974).

[4] L.J. Hall and S. Weinberg, Phys. Rev. D48, 979 (1993); see also A. Antaramian, L.J. Hall and A. Rasin, Phys. Rev. Lett. 69, 1871 (1992).

[5] For the most recent review see B. Winstein and L. Wolfenstein, Rev. of Mod. Phys. 65, 1113 (1993).

[6] J.M. Soares and L. Wolfenstein, Phys. Rev. D47, 1021 (1993). 